\title{
Lack of reduction in serum alpha-fetoprotein during treatment with direct antiviral agents predicts hepatocellular carcinoma development in a large cohort of patients with hepatitis $\mathrm{C}$ virus-related cirrhosis
}

\author{
Chiara Masetti ${ }^{1}$ (1) | Raffaella Lionetti ${ }^{2}$ Marinella Lupo $^{3}$ | Massimo Siciliano ${ }^{4}$ | \\ Valerio Giannelli ${ }^{5}$ | Francesca Romana Ponziani ${ }^{6}$ | Elisabetta Teti ${ }^{7}$ | \\ Chiara Dell'Unto $^{8}$ | Simona Francioso ${ }^{1}$ | Arianna Brega ${ }^{1}$ | Marzia Montalbano ${ }^{2}$ | \\ Ubaldo Visco-Comandini $^{2}$ | Chiara Taibi ${ }^{2}$ | Giovanni Galati ${ }^{8}$ | Umberto Vespasiani \\ Gentilucci $^{8}$ | Antonio Picardi ${ }^{8}$ | Massimo Andreoni ${ }^{7}$ | Maurizio Pompili ${ }^{6}$ | \\ Adriano M. Pellicelli ${ }^{5}$ | Gianpiero D'Offizi ${ }^{2}$ | Antonio Gasbarrini ${ }^{4}$ | \\ Adriano De Santis $^{3}$ | Mario Angelico ${ }^{1}$
}

${ }^{1}$ Liver and Transplant Unit, Tor Vergata

University Hospital, Rome, Italy

${ }^{2}$ Infectious Diseases-Hepatology, National Institute for Infectious Diseases Spallanzani, Rome, Italy

${ }^{3}$ Department of Clinical

Medicine, Gastroenterology Unit, Sapienza

University of Rome, Rome, Italy

${ }^{4}$ Gastroenterology Unit, Catholic University of Rome, Rome, Italy

${ }^{5}$ Liver Disease Unit, Department of Liver Transplantation, San Camillo Forlanini Hospital, Rome, Italy

${ }^{6}$ Internal Medicine, Gastroenterology and Hepatology, A. Gemelli Hospital, Rome, Italy

${ }^{7}$ Department of Infectious Disease, Tor Vergata University Hospital, Rome, Italy

${ }^{8}$ Internal Medicine and Hepatology Unit, University Campus Bio-Medico, Rome, Italy

Correspondence

Chiara Masetti, Liver and Transplant Unit, Tor Vergata University Hospital, Rome, Italy. Email: chiara.masetti12@gmail.com

\section{Summary}

Risk of hepatocellular carcinoma (HCC) in hepatitis $\mathrm{C}$ virus cirrhotic patients treated with direct-acting antiviral agents (DAA) is still debating. We investigated it in a large cohort. The cohort comprised 1045 cirrhotic patients who completed treatment with DAA, with a median follow-up of 17.3 months after end of treatment (EOT), including 943 patients without history of HCC and 102 previously treated for HCC. The majority were men (59.9\%), with compensated cirrhosis (88.8\%), genotype 1b (44.7\%). Univariate, multivariate analysis and Kaplan-Meier curves were performed to detect predictors of HCC in patients with and without reduction in alpha-fetoprotein (AFP) during treatment. SVR12 was 95.6\%. HCC developed in 95 (9.9\%), including 54 of $943(5.7 \%)$ occurrent and 41 of 102 (39\%) recurrent tumours. De novo were more often unifocal $(P=0.01)$ and curable $(P=0.03)$. AFP decreased from $16.1 \pm 36.2 \mathrm{mg} /$ $\mathrm{dL}$ (baseline) to $11.4 \pm 55 \mathrm{mg} / \mathrm{dL}$ (EOT). At univariate analysis, predictors were a previous HCC, older age, higher model for end-stage liver disease, prolonged INR, lower platelets, baseline and EOT AFP, virological failure and no reduction in AFP during treatment. Kaplan-Meier curves showed lower incidence of HCC in patients showing any reduction in AFP $(P=0.001)$. Those with AFP $<6 \mathrm{ng} / \mathrm{mL}$ had the lowest risk $(P=0.0002)$. At logistic regression, platelets $(P=0.009$, OR $0.99 \mathrm{Cl}$ : $0.99-1.00)$, previous HCC ( $P<0.000$ 01, OR: 10.76, 95\% Cl: 5.89-19.34) and no reduction in AFP during treatment $(P=0.0005$, OR: $2.98, \mathrm{Cl}: 1.60-5.54)$ were independent predictors of HCC. In conclusion, risk of HCC after DAA treatment remains substantial. It is higher 
among patients with previous HCC, low platelets and without reduction in AFP during treatment.

\section{KEYWORDS}

alpha-fetoprotein, direct-acting antiviral agents, hepatitis C, hepatocellular carcinoma

\section{1 | INTRODUCTION}

Newly developed or recurrent hepatocellular carcinoma (HCC) after hepatitis $C$ virus (HCV) eradication with oral direct-acting antiviral agents (DAA) has currently become a matter of debate in the hepatology community. After two different studies showed an unexpected high rate of HCC occurrence and recurrence after HCV eradication with DAA, ${ }^{1,2}$ a huge amount of data has been presented on this issue with conflicting results, making the question a big controversy. The most recent literature seems to deny a correlation between antiviral therapy and a higher risk of liver cancer. Kanwal et al ${ }^{3}$ showed that $\mathrm{HCV}$ eradication is associated with a significant reduction in the risk of subsequent HCC development, which remains, however, $1.82 \% / y$, thus justifying the need of continuous surveillance every 6 months in cirrhotic patients who achieve a sustained virological response (SVR). Similarly, loannou et $\mathrm{al}^{4}$ found a $71 \%$ reduction risk in $\mathrm{HCV}$ patients eradicated with DAA with an annual incidence of HCC of $1.97 \%$; risk remains relatively high $(3.25 \% / y)$ in cirrhotic patients with virological failure to treatment.

Several studies have investigated the risk factors associated with HCC onset in patients achieving SVR with interferon (IFN)-based regimens. Diabetes, low albumin levels, low platelet count, advanced age and high alcohol consumption have all been associated with higher risk of HCC in patients who attained SVR ${ }^{5-7}$ Less data exist, however, in patients in whom HCV was eradicated with DAAs. A Japanese study including 954 patients treated with both triple therapy or all-oral antiviral therapy showed that advanced liver fibrosis and $\mathrm{BMI}>23 \mathrm{~kg} / \mathrm{m}^{2}$ were independently correlated with hepatocarcinogenesis. ${ }^{8}$ In the study by Conti et al, ${ }^{2}$ Child-Pugh class, liver fibrosis and a previous history of $\mathrm{HCC}$ were all associated with an increased risk of liver cancer.

Alpha-fetoprotein (AFP) is widely used as a serological marker in patients at risk of HCC, although its diagnostic sensitivity is rather low, being serum levels elevated only in $60 \%$ of patients with HCC.? Moreover, an elevation of serum AFP can be observed also in patients with chronic HCV infection without $\mathrm{HCC}$ and has been associated with abnormal transaminases, advanced fibrosis and high model for end-stage liver disease (MELD) scores. ${ }^{10,11}$ Changes in AFP levels during IFN treatment have also been investigated. Two Japanese studies showed that an AFP reduction below $10 \mathrm{ng} / \mathrm{mL}$ after end of treatment (EOT) was associated with a lower subsequent risk of developing HCC. ${ }^{12,13}$ To our knowledge, little data exist about the predictive role of serum AFP in the setting of antiviral treatment with DAA. In a recently published study, a significant decrease in AFP levels was observed in cirrhotic patients achieving SVR with DAA,

\section{Key points}

- Hepatitis C virus-infected cirrhotic patients maintain a substantial residual risk of $\mathrm{HCC}$ even after treatment with DAA.

- The risk of HCC is much greater in patients who had a history of successfully treated HCC prior to treatment with DAA.

- Lack of reduction in AFP levels during treatment and even slightly elevated serum AFP levels at EOT are independently associated with a greater risk of either recurrent or occurrent HCC.

likely due to a reduction in liver inflammation. ${ }^{14}$ This suggests that in HCV-eradicated patients, persistently elevated AFP levels might be a more specific predictor of HCC.

The aim of this study was therefore to investigate HCC occurrence and recurrence in a large cohort of $\mathrm{HCV}$-infected cirrhotic patients who completed a full treatment course with DAA, identifying eventual risk factors for cancer development. We focused particularly on AFP trend during treatment in order to evaluate its predictive power after HCV eradication.

\section{2 | MATERIALS AND METHODS}

This observational cohort study included all HCV-infected cirrhotic patients consecutively treated with second-generation DAAs in 8 third-level units in Rome, between January 2015 and October 2017. The study was conducted according to the Declaration of Helsinki and received ethical approvement. Eligibility of individual patients for treatment of hepatitis $C$ with DAAs was assessed according to the prioritization criteria established by the Italian National Drug Agency (AIFA). Antiviral treatment was provided according to the most recent guidelines of the European Association for the Study of the Liver (EASL) at the time of treatment. ${ }^{15}$ Whenever alternative treatment options were available for the same indication, the choice of therapy was left at the clinician's discretion. Patients coinfected with HBV or HIV were excluded from this study.

Before starting antiviral therapy, all patients were assessed in order to define the stage of liver disease and to exclude the presence of HCC, using clinical evaluation, transient elastography and abdominal ultrasonography. The diagnosis of liver cirrhosis was based on 
the evidence of liver with nodular surface and coarse echo pattern at ultrasonography and/or the presence of clinical features of portal hypertension (eg, ascites, splenomegaly and the presence of oesophageal varices), and/or a liver stiffness $\geq 13 \mathrm{kPa}$ at transient elastography. The diagnosis of HCC was based on the detection of one or more focal lesions with at least two imaging techniques as computerized tomography (CT) and magnetic resonance imaging (MRI), or with one imaging technique demonstrating a typical radiological pattern (arterial hypervascularity and early/late venous phase washout). In patients with a previous history of HCC, there was no evidence of active neoplastic disease before starting antiviral therapy, based on ultrasonographic surveillance every 3 months. The response to DAA therapy was assessed by the quantitative assay of HCV-RNA in serum using real-time protein chain reaction (PCR) with a limit of detection of $12 \mathrm{lU} / \mathrm{mL}$ (Abbott Real-Time PCR). The definitions of EOT and of SVR were consistent with current European guidelines. ${ }^{15}$

All data from the eight units participating in the study were collected into an electronic database, which comprised anthropometric findings, stadiation of liver disease and portal hypertension, treatment schedule, genotype, viral load, transaminases and liver function tests at baseline, AFP levels before and after treatment. Data about HCC de novo or recurrent (month of occurrence since EOT, number and size of nodules, the presence of vascular invasion, and inclusion in Milan criteria, treatment performed) were also recorded.

By the end of October 2017, the database comprised 1045 consecutive patients with HCV-related advanced liver disease and no evidence of HCC, who completed treatment with DAA and had a minimum follow-up of 6 months after EOT. Surveillance for HCC after the end of therapy was conducted with ultrasound examination every 6 months in cirrhotic patients without a previous history of HCC and every 3 months in patients who already had an HCC. If one or more suspected lesion were detected, patients underwent a second-level imaging technique as CT or MRI. Any decline in AFP levels was considered significant in the subsequent analysis.

Statistical analysis was performed by NCSS 10 statistical software (NCSS, East Kaysville, UT, USA). Continuous variables were expressed as means \pm standard deviation, while categorical variables as percentages. Differences between patients who developed or not HCC were analysed using Chi-square for categorical variables and Student's $t$ test for continuous variables. Kaplan-Meier's curves were used to estimate the cumulative incidence of HCC and the logrank test was used to compare time-to-event curves. Independent predictors of incident HCC were analysed by stepwise Cox regression analysis. Statistical significance was taken as $P$-value $<0.05$.

\section{3 | RESULTS}

The demographic, clinical, virological and biochemical characteristics of the 1045 patients included in the study are summarized in Table 1. The majority of patients were men (59.9\%), mean age was $63.7 \pm 11.6$ years and the most frequent $\mathrm{HCV}$ genotype was $1 \mathrm{~b}$ (44.7\%). Most of the patients (88.8\%), when starting DAA treatment, had compensated cirrhosis with low median MELD score $(9 \pm 2.6)$; conversely, in 113 patients (11.2\%), cirrhosis was classified as ChildPugh class B or C. Liver stiffness as measured by transient elastography averaged $22.7 \pm 11.7 \mathrm{KPa}$, proving advanced fibrosis. No clinically significant portal hypertension was found in $67.1 \%$ of patients, while $29 \%$ of the population had small F1 oesophageal varices and only $3.9 \%$ had large F2-F3 varices. One hundred and two patients had a previous history of HCC which had been successfully treated before starting DAA treatment. All patients included in the present analysis had completed a full DAA treatment course, which in most cases (85.7\%) included sofosbuvir as a backbone antiviral therapy, and had a minimum follow-up of 6 months after the EOT. SVR was obtained in 995 patients (95.6\%).

Alpha-fetoprotein values were assayed at baseline and at EOT: in the vast majority of patients (85.8\%), AFP levels decreased after treatment, the mean levels falling from $16.1 \pm 36.2 \mathrm{ng} / \mathrm{mL}$ at baseline to $11.4 \pm 55.0 \mathrm{ng} / \mathrm{mL}$ at EOT, respectively $(P=0.006)$. Reduction in AFP levels during treatment remained statistically significant in patient without a previous history of $\mathrm{HCC}(P<0.00001)$ but not in those with a previous $\operatorname{HCC}(P=0.24$; Figure 1$)$.

No relation was observed between the entity of decrease in AFP levels ( $\triangle \mathrm{AFP})$ and the subsequent development of HCC ( $P 0.26)$.

During the study period (the current median follow-up after reaching EOT is $17.3 \pm 7.2$ months), 95 patients (9.9\%) had an incident HCC. As expected, incidence was higher in patients with recurrent (41 of $102,39 \%$ ) than occurrent tumours (54 of $943,5.7 \%$ ). Number, size, vascular invasion and type of treatment of newly diagnosed HCC are summarized in Table 2 . In patients who already had a history of HCC, the type of treatment refers to the recurrent HCC. Median time from EOT to HCC diagnosis was 9.6 and 8.1 months for occurrent and recurrent HCC, respectively $(P=0.26)$. The majority of patients $(56.8 \%)$ presented with a single focal lesion; the average diameter of the largest neoplastic lesion was $21.8 \pm 13.1 \mathrm{~mm}$. Only five patients presented with vascular invasion at diagnosis and extrahepatic tumour spread was not observed. Eighty-three percentage of patients were within Milan criteria, and $56.3 \%$ achieved curative treatments. According to Kaplan-Meier curves (Figure S1), the 12 -month incidence rates were $4.4 \%$ and $28.7 \%$ for de novo and recurrent HCC, respectively (log rank; $P=0.00001$ ).

At univariate analysis, patients who developed an incident HCC during the study period were older $(P=0.005)$, had more frequently a history of previous HCC $(<0.00001)$, presented with a more advanced disease with higher MELD score $(P=0.0002)$, more prolonged INR $(P=0.02)$, a higher total bilirubin $(P=0.01)$ and a lower platelet count $(P=0.03)$; moreover, they presented significantly lower rates of SVR $(P<0.00001)$. Finally, in patients who developed a subsequent HCC, we observed higher levels of AFP both at baseline and at EOT ( $P=0.00006$ and $P<0.00001$, respectively) and a higher rate of AFP level $>6 \mathrm{ng} / \mathrm{mL}$ at EOT; they also had reduction in AFP levels during treatment much less frequently compared to patients who did not develop HCC. Other previously suggested risk factors such as high body mass index or male gender failed to show any association with the development of HCC. In addition, HCV viral load, 
TA B LE 1 Demographic, clinical, virological and biochemical features of patients without or with incident HCC after EOT and of the whole study population

\begin{tabular}{|c|c|c|c|c|}
\hline Variable & $\begin{array}{l}\text { Whole study } \\
\text { population }(n=1045)\end{array}$ & $\begin{array}{l}\text { Patients without incident HCC } \\
(\mathrm{n}=950)\end{array}$ & $\begin{array}{l}\text { Patients with incident HCC } \\
(\mathrm{n}=95)\end{array}$ & P-values \\
\hline Age (y) & $63.7 \pm 11.6$ & $63.3 \pm 11.6$ & $66.8 \pm 10.9$ & 0.005 \\
\hline Male gender, \% & 59.9 & 59.1 & 67.6 & 0.11 \\
\hline Body mass index $\left(\mathrm{kg} / \mathrm{m}^{2}\right)$ & $25.6 \pm 4.2$ & $25.7 \pm 4.3$ & $24.9 \pm 3.8$ & 0.08 \\
\hline Liver stiffness (KPa) & $22.7 \pm 11.5$ & $22.7 \pm 11.6$ & $22.7 \pm 10.0$ & 0.95 \\
\hline \multicolumn{5}{|l|}{ Oesophageal varices, \% } \\
\hline FO & 67.1 & 68.1 & 57.1 & \multirow[t]{3}{*}{0.09} \\
\hline F1 & 29.0 & 28.0 & 39.3 & \\
\hline F2-F3 & 3.9 & 3.9 & 3.6 & \\
\hline \multicolumn{5}{|l|}{ Child-Pugh stage, $\%$} \\
\hline A & 88.8 & 89.5 & 82.6 & \multirow[t]{3}{*}{0.12} \\
\hline B & 10.7 & 10.1 & 16.3 & \\
\hline $\mathrm{C}$ & 0.5 & 0.4 & 0.1 & \\
\hline MELD score & $9.0 \pm 2.6$ & $8.9 \pm 2.6$ & $10.1 \pm 3.3$ & 0.0002 \\
\hline Previous HCC, \% & 9.8 & 6.3 & 44.6 & $<0.00001$ \\
\hline HCV-RNA (IU/mL) & $1.59 \pm 3.40 \times 10^{6}$ & $1.62 \pm 3.53 \times 10^{6}$ & $1.25 \pm 1.67 \times 10^{6}$ & 0.30 \\
\hline \multicolumn{5}{|l|}{ Viral genotype,\% } \\
\hline $1 \mathrm{a}$ & 16.6 & 17.5 & 9.4 & \multirow[t]{5}{*}{0.24} \\
\hline $1 \mathrm{~b}$ & 44.7 & 43.6 & 54.7 & \\
\hline 2 & 15.7 & 16.0 & 12.7 & \\
\hline 3 & 15.1 & 14.8 & 17.9 & \\
\hline 4 & 7.9 & 8.1 & 5.3 & \\
\hline Sustained virological response, \% & 95.6 & 96.9 & 82.1 & $<0.00001$ \\
\hline Median follow-up (mo) & $17.3 \pm 7.2$ & $18.2 \pm 6.6$ & $8.9 \pm 6.6$ & $<0.000001$ \\
\hline ALT (IU/L) & $84.5 \pm 64.1$ & $85.1 \pm 63.7$ & $78.7 \pm 68.3$ & 0.36 \\
\hline Platelets (units $/ \mu \mathrm{L}$ ) & $127 \pm 68 \times 10^{3}$ & $129 \pm 68 \times 10^{3}$ & $113 \pm 65 \times 10^{3}$ & 0.03 \\
\hline Total bilirubin (mg/dL) & $1.05 \pm 0.72$ & $1.03 \pm 0.70$ & $1.22 \pm 0.88$ & 0.01 \\
\hline INR & $1.17 \pm 0.28$ & $1.17 \pm 0.29$ & $1.24 \pm 0.27$ & 0.02 \\
\hline Total cholesterol (mg/dL) & $145 \pm 36$ & $146 \pm 35$ & $137 \pm 41$ & 0.05 \\
\hline eGFR (mL/min) & $91.0 \pm 28.8$ & $91.5 \pm 28.9$ & $85.9 \pm 27.3$ & 0.11 \\
\hline Baseline AFP (ng/mL) & $16.1 \pm 36.2$ & $14.5 \pm 28.0$ & $30.9 \pm 77.1$ & 0.00006 \\
\hline AFP at EOT (ng/mL) & $11.4 \pm 55.0$ & $6.1 \pm 10.1$ & $49.1 \pm 149.6$ & $<0.00001$ \\
\hline$\Delta \mathrm{AFP}(\mathrm{ng} / \mathrm{mL})$ & $13.2 \pm 29.6$ & $12.7 \pm 30.0$ & $17.3 \pm 25.7$ & 0.26 \\
\hline AFP reduction, $\%$ & 85.8 & 87.8 & 72.1 & 0.0007 \\
\hline AFP at EOT $\geq 6 \mathrm{ng} / \mathrm{mL}$ rate, $\%$ & 32.6 & 30.0 & 51.1 & 0.00007 \\
\hline \multicolumn{5}{|l|}{ DAA treatment schedue, $\%$} \\
\hline SOF alone & 18.2 & 18.2 & 18.1 & \multirow[t]{7}{*}{0.77} \\
\hline SOF + SMV & 17.5 & 17.1 & 22.3 & \\
\hline $\mathrm{SOF}+\mathrm{DCV}$ & 16.9 & 16.7 & 18.1 & \\
\hline SOF + LDV & 31.5 & 32.0 & 26.6 & \\
\hline SOF + VEL & 1.6 & 1.7 & 1.1 & \\
\hline$P A R+O M V+R T V$ & 1.4 & 1.5 & 0 & \\
\hline$P A R+O M V+R T V+D A S$ & 12.9 & 12.8 & 13.8 & \\
\hline
\end{tabular}

AFP, alpha-fetoprotein; ALT, alanine amino-transferase; DAA, direct-acting antivirals; DAS, dasabuvir; DCV, daclatasvir; eGFR, estimated glomerular filtration rate; EOT, end of treatment; HCC, hepatocellular carcinoma; HCV, hepatitis C virus; INR, international normalized ratio; LDV, ledipasvir; MELD, model for end-stage liver disease; OMV, ombitasvir; PAR, paritaprevir; RTV, ritonavir; SMV, simeprevir; SOF, sofosbuvir; SVR, sustained virological response; VEL, velpatasvir.

Statistically significant values are in bold. 
$\mathrm{HCV}$ genotype and the DAA regimen were also unrelated to the occurrence of HCC.

Figure 2 shows the Kaplan-Meier estimates of the incidence of $\mathrm{HCC}$ according to the reduction in AFP levels during treatment with DAA. Patients without reduction in AFP levels during treatment had a higher risk of HCC development $(P=0.001)$. Moreover, patients who maintain AFP levels $>6 \mathrm{ng} / \mathrm{mL}$ at EOT had a much greater incidence of $\mathrm{HCC}$ compared to those with AFP $\leq 6 \mathrm{ng} / \mathrm{mL}(P=0.0002$, Figure 3 ).

A multivariable logistic regression analysis was performed in order to assess the independent predictors of HCC development after EOT. All the variables found to have a significant association, or even a trend, with $\mathrm{HCC}$ formation at univariate analysis, was tested in the logistic regression analysis. In the final model, only three variables emerged as significant independent predictors of $\mathrm{HCC}$

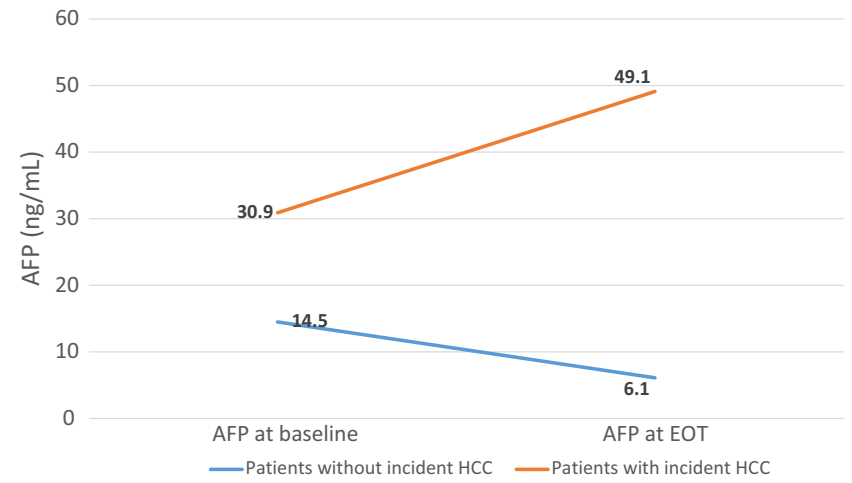

FIGURE 1 Variations in AFP levels from baseline to EOT in patients with and without incident HCC. AFP, alpha-fetoprotein; EOT, end of treatment; HCC, hepatocellular carcinoma formation after EOT: a history of previous HCC $(P<0.00001$, OR: 10.76, 95\% Cl: 5.89-19.34), platelet count at baseline ( $P=0.009$, OR $0.99 \mathrm{Cl}: 0.99-1.00)$ and lack of reduction in AFP levels during treatment with DAA ( $P=0.0005$, OR: 2.98, Cl: 1.60-5.54; Table 3).

\section{4 | DISCUSSION}

Hepatocellular carcinoma is one of the most frequent and severe complications in patients with advanced liver disease. In HCVinfected patients, achievement of SVR has been clearly correlated with a reduction in the lifetime risk of developing $\mathrm{HCC} .{ }^{16}$ Recent literature, however, shows that cirrhotic patients are still at risk of developing $\mathrm{HCC}$ even after viral clearance, thus requiring continuous and regular surveillance. The residual risk of HCC has been estimated in a large cohort of HCV-infected patients treated with IFN with or without ribavirin, being $0.33 \% / y$ in the whole cohort and $1.39 \% / y$ in a subanalysis including only cirrhotic patients. ${ }^{17}$

The molecular mechanisms underlying the transition from cirrhosis to HCC development are still poorly understood. Several viral factors are thought to be implicated in hepatocarcinogenesis, possibly by altering specific pathways involved in either oxidative stress, lipid metabolism, angiogenesis, cell proliferation or apoptosis. Indeed, the trigger to carcinogenesis may possibly be only indirect, being represented by the virus-induced chronic persistent inflammation in the liver. ${ }^{18,19}$ It has been then speculated that DAA may accelerate the development of $\mathrm{HCC}$ due to the rapid reduction in HCV-RNA production and the subsequent disruption of immune surveillance, leading to the escape of neoplastic clones. ${ }^{1}$

TAB LE 2 Clinical characteristics of occurrent and recurrent HCC

\begin{tabular}{|c|c|c|c|c|}
\hline Variable & $\begin{array}{l}\text { Patients with occurrent HCC } \\
(n=54)\end{array}$ & $\begin{array}{l}\text { Patients with recurrent } \mathrm{HCC} \\
(\mathrm{n}=41)\end{array}$ & $P$-values & $\begin{array}{l}\text { Whole study } \\
\text { population }(n=95)\end{array}$ \\
\hline \multicolumn{5}{|l|}{ Number of lesions, \% } \\
\hline 2 & 29.6 & 34.1 & \multirow{2}{*}{0.01} & 31.6 \\
\hline 3 or more & 3.8 & 22 & & 11.6 \\
\hline Vascular invasion, \% & 3.7 & 7.3 & 0.43 & 5.3 \\
\hline Milan criteria in, \% & 83.3 & 82.5 & 0.49 & 83.0 \\
\hline \multicolumn{5}{|l|}{ Type of treatment, \% } \\
\hline OLT & 8.3 & 13.2 & \multirow[t]{4}{*}{0.03} & 10.3 \\
\hline Resection & 30.6 & 7.9 & & 20.8 \\
\hline TARE & 0 & 5.3 & & 2.4 \\
\hline No treatment & 14.3 & 2.6 & & 9.2 \\
\hline
\end{tabular}

HCC, hepatocellular carcinoma; OLT, orthotopic liver transplant; RFTA, radiofrequency thermal ablation; TACE, transarterial chemoembolization; TARE, transarterial radioembolization.

Statistically significant values are in bold. 


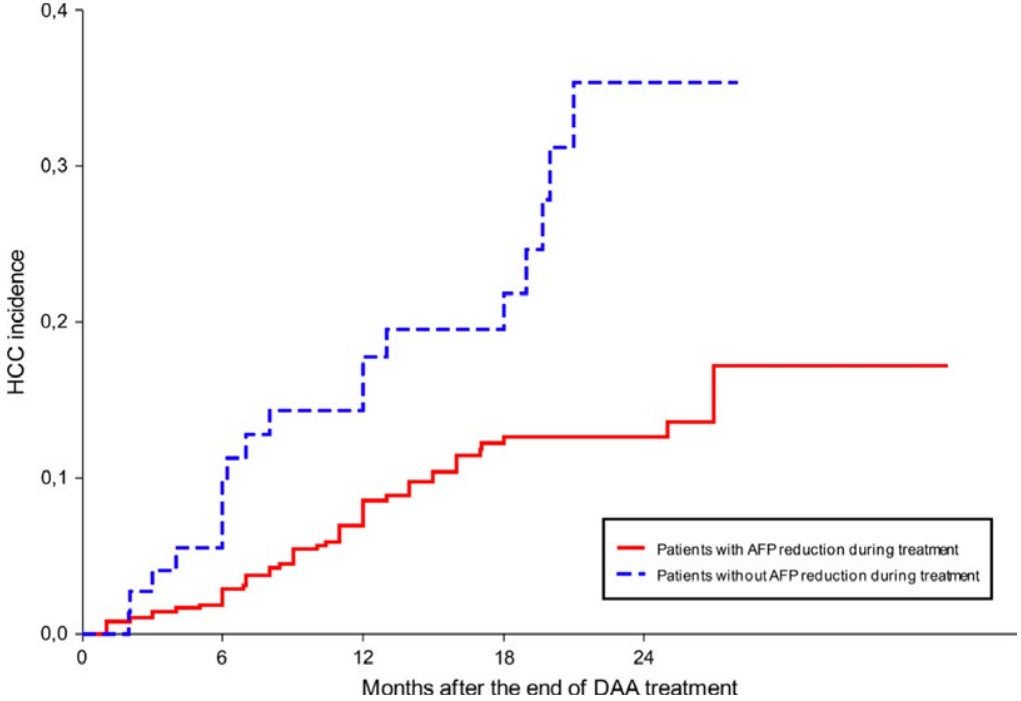

Number of patients at risk

\begin{tabular}{lllllr} 
AFP reduction & 515 & 476 & 367 & 250 & 122 \\
\hline
\end{tabular}

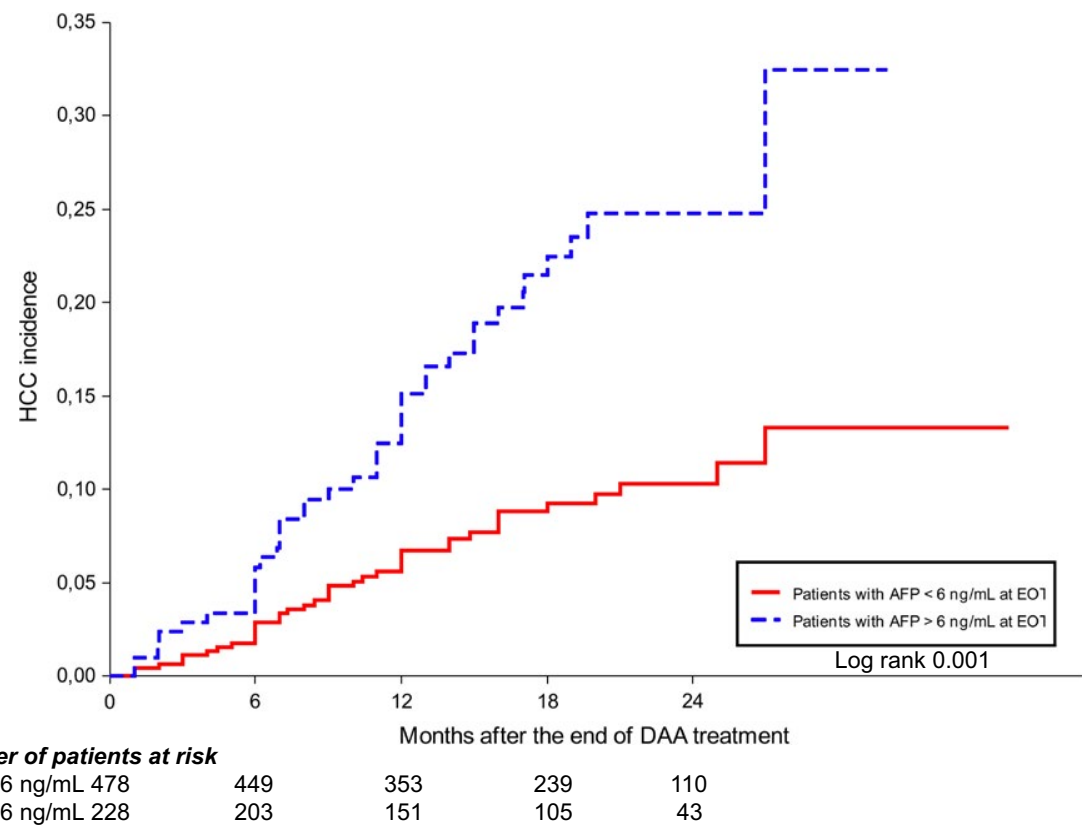

FIGURE 2 Incidence of HCC in the study population according to reduction in AFP levels during treatment with DAA (Kaplan-Meier's analysis). AFP, alphafetoprotein; DAA, direct-acting antiviral agents; HCC, hepatocellular carcinoma
FIGURE 3 Incidence of HCC in the study population according to AFP cut-off level of $6 \mathrm{ng} / \mathrm{mL}$ at EOT (Kaplan-Meier's analysis). AFP, alpha-fetoprotein; EOT, end of treatment; HCC, hepatocellular carcinoma
The present study included a large multicentre cohort of consecutive HCV-infected cirrhotic patients who completed a treatment course with DAA. The study cohort comprised a subgroup of patients (9.8\%) who had a previous history of HCC and were tumourfree when starting treatment. SVR rates were high (95.6\%) and did not differ between patients with or without a previous history of HCC ( $P=0.35)$, a finding well in keeping with available data in the cirrhotic population. ${ }^{20}$ This result is even more impressive considering that almost one in five patients received a therapy with sofosbuvir alone, which is now considered as suboptimal.

During a median follow-up of 17.3 months after EOT, 95 patients (9.9\%) developed HCC. This figure is consistently higher than that observed in cirrhotic patients who achieved SVR and even higher than in those with ongoing HCV infection. ${ }^{21}$ Notably, HCC incidence rates differ significantly between patients who achieved SVR and those who relapsed after EOT $(P<0.000001)$, as already shown by loannou et al. ${ }^{4}$ In agreement with Conti et al, ${ }^{2}$ the 12-month risk of recurrent $\mathrm{HCC}$ was much higher (39\%) compared to the risk of de novo HCC (5.7\%).

Predictive risk factors associated with an increased risk of carcinogenesis in patients achieving SVR with DAA have not yet been elucidated. In this setting, the role of AFP in estimating the residual risk is unclear. Recent recommendations of the American Association for the Study of the Liver (AASLD) ${ }^{22}$ indicate that HCC surveillance should be based on imaging procedures at 6-month intervals after the achievement of SVR in cirrhotic patients, but 
TAB LE 3 Logistic regression analysis of predictors of incident HCC in cirrhotic patients after the end of treatment with DAA

\begin{tabular}{|c|c|c|c|c|}
\hline $\begin{array}{l}\text { Independent } \\
\text { variable }\end{array}$ & $P$ & Hazard ratio & $\begin{array}{l}\text { Lower } 95 \% \\
\text { confidence limit }\end{array}$ & $\begin{array}{l}\text { Upper } 95 \% \\
\text { confidence limit }\end{array}$ \\
\hline $\begin{array}{l}\text { History of } \\
\text { previous HCC }\end{array}$ & $<0.000001$ & 10.67 & 5.89 & 19.34 \\
\hline $\begin{array}{l}\text { Platelet count at } \\
\text { baseline }\end{array}$ & 0.009 & 0.99 & 0.99 & 1.00 \\
\hline $\begin{array}{l}\text { No reduction in } \\
\text { AFP levels }\end{array}$ & 0.0005 & 2.98 & 1.60 & 5.54 \\
\hline
\end{tabular}

AFP, alpha-fetoprotein; DAA, direct-acting antiviral agents; HCC, hepatocellular carcinoma. also suggest that the role of AFP determination, though currently considered only adjunctive or optional, should be better investigated in the post-SVR surveillance. In the study by Conti et al, ${ }^{2}$ AFP levels at baseline were not significantly different between patients with or without HCC development after DAA treatment. In a recent Japanese study, Akuta et $a^{8}{ }^{8}$ found that AFP levels at baseline above $10 \mathrm{ng} / \mathrm{mL}$ significantly correlated with hepatocarcinogenesis at univariate analysis, yet this result was not confirmed at multivariate analysis. To our knowledge, predictive role of AFP levels measured at EOT with DAA has not yet been investigated. In the present study, multivariate analysis showed that the absence of reduction in AFP levels during treatment was independently associated with HCC development and that the risk of HCC development is significantly higher in patients who maintain even slightly elevated AFP at EOT. Notably, AFP levels decreased significantly from baseline to the EOT, in parallel with the normalization of alanine amino-transferase (ALT) and the rapid DAA-induced viral suppression, suggesting that baseline AFP levels may reflect in part the extent of liver inflammation. Indeed, this possibility has been already reported in the setting of chronic HBV infection during treatment with nucleoside analogs. In fact, in a large cohort of patients with HBV-related cirrhosis treated with entecavir, ${ }^{23}$ AFP levels were found to decrease after viral suppression, together with reduced liver inflammation. On the other hand, patients who maintained elevated AFP levels, even after ALT normalization, were more prone to develop HCC in the follow-up. Interestingly, in this study, the authors found that an AFP cut-off value as low as $6 \mathrm{ng} / \mathrm{mL}$ had the greatest sensitivity in predicting $\mathrm{HCC}$ occurrence. We sought here to investigate the predictive role of AFP measured at EOT in HCV-infected cirrhotic patients treated with DAA, when HCV-induced liver inflammation had presumably been blunted, thus no longer behaving as a potential confounder. As clearly shown by the Kaplan-Meier curves, the risk of developing $\mathrm{HCC}$ was remarkably higher in patients with AFP levels above $6 \mathrm{ng} / \mathrm{mL}$ compared to those with AFP $\leq 6 \mathrm{ng}$ / $\mathrm{mL}$, and this was already found after the initial months after EOT. Notably, this occurred both in patients with and without a previous history of HCC. The curves were also similar after excluding patients with virological failure, in whom inflammation due to persistent virological activity could influence AFP levels. Therefore, as already reported in the HBV setting, the simple determination of AFP levels in HCV-infected cirrhotic patients at completion of a DAA treatment course allows to identify a subgroup of patients at greater risk of cancer, characterized by only minimally elevated AFP. It is tempting to speculate that these patients may require a more strict surveillance for $\mathrm{HCC}$ than currently recommended.

Our study has several limitations. First, more data, hopefully from controlled studies, are needed to judge the actual risk of developing $\mathrm{HCC}$ in patients who achieve HCV eradication with DAA, and a longer period of follow-up would definitely be necessary to draw definitive conclusions. Second, likewise the other available reports, our study missed a control group, which does not exclude the possibility of a patient selection bias. Indeed, the current availability of safe, easy-to-use and potent DAAs allows to treat with IFN-free short schedules patients who were untreatable until recently, who might be intrinsically more prone to develop cancer. Third, understanding the carcinogenesis process in this setting would certainly require specific studies collecting pathological and immunological data to investigate tumour biology and the alterations in the immune system occurring after the abrupt HCV elimination.

In conclusion, in this multicentre study of a large cohort of HCV-infected cirrhotic patients who complete a DAA treatment course, the residual risk of $\mathrm{HCC}$ was substantial, regardless that the vast majority of patients attained SVR. Lack of reduction in AFP levels during treatment, platelet count at baseline and a history of previous HCC were significant and independent predictors of early HCC development. Moreover, persistence of even minimally elevated AFP levels at EOT might represent an additional risk factor.

\section{CONFLICT OF INTEREST}

All the authors have given substantial contribution to the completion of this work and have seen and approved the text in the current version. None reported a conflict of interest with respect to this manuscript.

\section{ORCID}

Chiara Masetti iD http://orcid.org/0000-0002-1689-4876

\section{REFERENCES}

1. Reig M, Mariño Z, Perelló C, et al. Unexpected early tumor recurrence in patients with hepatitis $\mathrm{C}$ virus -related hepatocellular 
carcinoma undergoing interferon-free therapy: a note of caution. J Hepatol. 2016;65(4):719-726.

2. Conti F, Buonfiglioli F, Scuteri A, et al. Early occurrence and recurrence of hepatocellularcarcinoma in HCV-related cirrhosistreated with directactingantivirals. J Hepatol. 2016;65(4):727-733.

3. Kanwal F, Kramer J, Asch SCM, Cao Y, El-Serag HB. Risk of hepatocellular cancer in HCV patients treated with direct acting antiviral agents. Gastroenterology. 2017;153(4):996-1005.

4. Ioannou GN, Green PK, Berry K. HCV eradication induced by direct-acting antiviral agents reduces the risk of hepatocellular carcinoma. J Hepatol. 2018;68(1):25-32.

5. Hedenstierna M, Nangarhari A, Weiland O, Aleman S. Diabetes and cirrhosis are risk factors for hepatocellular carcinoma after successful treatment of chronic hepatitis C. Clin Infect Dis. 2016;63(6):723-729.

6. Ikeda M, Fujiyama S, Tanaka M, et al. Risk factors for development of hepatocellular carcinoma in patients with chronic hepatitis C after sustained response to interferon. J Gastroenterol. 2005;40(2):148-156.

7. Tokita H, Fukui $\mathrm{H}$, Tanaka A, et al. Risk factors for the development of hepatocellular carcinoma among patients with chronic hepatitis C who achieved a sustained virological response to interferon therapy. Clin Gastroenterol Hepatol. 2005;20(5):752-758.

8. Akuta N, Kobayashi M, Suzuki F, et al. Liver fibrosis and body mass index predict hepatocarcinogenesis following eradication of hepatitis C virus RNA by direct-acting antivirals. Oncology. 2016;91(6):341-347.

9. Bruix J, Reig M, Sherman M. Evidence-based diagnosis, staging and treatment of patients with hepatocellular carcinoma. Gastroenterology. 2016;150(4):835-853

10. Hu KQ, Kyulo NL, Lim N, Elhazin B, Hillebrand DJ, Bock T. Clinical significance of elevated alpha-fetoprotein (AFP) in patients with chronic hepatitis $\mathrm{C}$, but not hepatocellular carcinoma. Am J Gastroenterol. 2004;99(5):860-865.

11. Richardson P, Duan Z, Kramer J, Davila JA, Tyson GL, ElSerag HB. Determinants of serum alpha-fetoprotein levels in hepatitis C-infected patients. Clin Gastroenterol Hepatol. 2012;10(4):428-433.

12. Tamura Y, Yamagiwa S, Aoki Y, et al. Serum alpha-fetoprotein levels during and after interferon therapy and the development of hepatocellular carcinoma in patients with chronic hepatitis C. Dig Dis Sci. 2009;54:2530-2537.

13. Osaki $\mathrm{Y}$, Ueda $\mathrm{Y}$, Marusawa $\mathrm{H}$, et al. Decrease in alpha-fetoprotein levels predicts reduced incidence of hepatocellular carcinoma in patients with hepatitis $C$ virus infection receiving interferon therapy; a single-center study. J Gastroenterol. 2012;47:444-451.
14. Nguyen K, Jimenez $M$, Moghadam N, et al. Decrease of alphafetoprotein in patients with cirrhosis treated with direct-acting antivirals. J Clin Transl Hepatol. 2017;5(1):43-49.

15. European Association for the Study of the Liver. EASL recommendations on treatment of hepatitis C 2016. J Hepatol. 2017;66(1):153-194.

16. Pearlman LB, Traub N. Sustained virological response to antiviral therapy for chronic hepatitis $C$ virus infection: a cure and so much more. Clin Infect Dis. 2011;52(7):889-900.

17. El-Serag HB, Kanwal F, Richardson P, Kramer J. Risk of hepatocellular carcinoma after sustained virological response in veterans with hepatitis C virus infection. Hepatology. 2016;64(1):130-137.

18. Vescovo T, Refolo G, Vitagliano G, Fimia GM, Piacentini M. Molecular mechanisms of hepatitis $C$ virus-induced hepatocellular carcinoma. Clin Microbiol Infect. 2016;22:853-861.

19. Bandiera S, Billie Bian C, Hoshida Y, Baumert TF, Zeisel MB. Chronic hepatitis $C$ virus infection and pathogenesis of hepatocellular carcinoma. Curr Opin Virol. 2016;20:99-105.

20. Toshikuni N. Therapy with direct-acting antiviral agents for hepatitis C-related cirrhosis. Gut Liv. 2017;11(3):335-348.

21. El-Serag HB, Rudolph KL. Hepatocellular carcinoma: epidemiology and molecular carcinogenesis. Gastroenterology. 2007;132(7):2557-2576.

22. Heimbach J, Kulik M, Finn R. AASLD guidelines for the treatment of hepatocellular carcinoma. Hepatology. 2018;67(1):358-380.

23. Wong GL, Chan HL, Tse YK, et al. On treatment alpha-fetoprotein is a specific tumor marker for hepatocellular carcinoma in patients with chronic hepatitis $\mathrm{B}$ receiving entecavir. Hepatology. 2014;59(3):986-995.

\section{SUPPORTING INFORMATION}

Additional supporting information may be found online in the Supporting Information section at the end of the article.

How to cite this article: Masetti C, Lionetti R, Lupo M, et al. Lack of reduction in serum alpha-fetoprotein during treatment with direct antiviral agents predicts hepatocellular carcinoma development in a large cohort of patients with hepatitis C virus-related cirrhosis. J Viral Hepat. 2018;00:1-8. https://doi.org/10.1111/jvh.12982 